$$
\text { s/31/98 }
$$

DEVELOPMENT OF A PHENOMENOLOGICAL MODEL FOR COAL SLURRY ATOMIZATION

FINAL REPORT FOR DOE GRANT NO DE-FG22-94PC94119

SUBMITTED BY

DR.JOHN DOOHER*

CHAIRMAN PHYSICS DEPARTMENT

ADELPHI UNIVERSITY

GARDEN CITY, NY 11530

(516) 877-4883

(516) 877-4887 (FAX)

DOOHER@ADELPHI.EDU

*YING OF BALQUHAIN LAUREATE

Scanned 


\section{DISCLAIMER}

This report was prepared as an account of work sponsored by an agency of the United States Government. Neither the United States Government nor any agency thereof, nor any of their employees, makes any warranty, express or implied, or assumes any legal liability or responsibility for the accuracy, completeness, or usefulness of any information, apparatus, product or process disclosed, or represents that its use would not infringe privately owned rights. Reference herein to any specific commercial product, process or service by trade name, trademark, manufacturer, or otherwise does not necessarily constitute or imply its endorsement, recommendation, or favoring by the United States Government or any agency thereof. The views and opinions of authors expressed herein do not necessarily state or reflect those of the United States Government or any agency thereof. 
CONTENTS

PAGE TOPIC

4 ABSTRACT

$5 \quad$ 1.0 BACKROUND AND INTRODUCTION

$15 \quad 2.0 \mathrm{METHODOLOGY}$

$25 \quad 3.0$ RESULTS

26 4.0 THEORETICAL DISCUSSION

$45 \quad 5.0$ CONCLUSIONS

47 REFERENCES 


\title{
DEVELOPMENT OF A PHENOMENOLOGICAL MODEL FOR COAL SLURRY ATOMIZATION
}

FINAL REPORT FOR DOE GRANT NO DE-FG22-94PC94119

\begin{abstract}
This report presents the results of an investigation of theoretical models of atomization which can be used for a phenomenological theory for coal slurry atomization. This investigation was conducted in three areas. An intensive analysis of the rheological properties of coal slurry fuels was performed including viscosity as a function of shear rate, the extensional viscosity, and the viscoelastic properties. In order to evaluate atomization over a sufficiently wide range of rheological properties simulated fluids consisting of corn syrup and water and baseline Newtonian fluids were studied. Another area was the atomization of both coal slurries and simulated fluids under a variety of spray conditions using a Malvern Size Analyzer. Three basic theoretical models were analyzed to determine the best approach to characterizing these complex fluids. One model was a linearized Navier Stokes equation for a cylindrical fluid stream breaking up into drops under the impact of a high velocity air stream. A second model was a collisional model by which the collision of the air stream and the fluid stream produced droplets. Energy and momentum conservation were used to derive relationships between the drop size and the relevant physical parameters. A third model studied was a statistical model using a Boltzmann-type transport equation for the propagation of drops under the interactions of a high velocity airstream. The effects of drop coalescence and breakup are incorporated into this model. By comparing the various theoretical models with the atomization data and the rheological data a phenomenological model was constructed which correctly predicted the trends of the Sauter mean Diameter as a function of air/fuel ratio, rheological properties and spray angle. An effective viscosity was defined which included the effects of viscous losses, extensional properties, and viscoelastic properties. In addition, the effects of yield point were incorporated and shown to be important in predicting atomization properties.
\end{abstract}




\subsection{BACKGROUND AND INTRODUCTION}

Highly concentrated suspensions of coal particles in water or alternate fluids appear to have a wide range of applications for energy production. Coal slurry fuels have applications for boiler furnaces and internal combustion engines. Since most coal cleaning processes produce coal slurries, it is natural to utilize dense suspensions for transportation and power generation. As a method of utilizing coal fines, coal slurries provide a viable approach. For successful commercial utilization of coal slurries, it is important that a thorough understanding of their properties be developed and applied for a basis for engineering and design for a wide variety of coal properties and slurry specifications.

For enhanced implementation of coal slurry fuel technology, an understanding of coal slurry atomization as a function of coal and slurry properties for specific mechanical configurations of nozzle atomizers has been developed in this program. For fuel oil combustion, well-defined correlations exist relating atomization properties, such as Sauter mean diameter, to the physical properties of the oil, i.e. viscosity. However, no such relationships existed for coal slurries. Unlike oils, which are essentially Newtonian fluids fully characterized by a few basic physical parameters, such as viscosity, density, and surface tension, coal slurries are complex structures with large numbers of internal degrees of freedom and are usually non-Newtonian. In order to examine some of the difficulties in modeling slurry atomization, it is useful to review work done in this field.

When coal slurries were first being considered as an alternate boiler-furnace fuel to oil, the importance of atomization had not been clearly delineated. Since a coal water fuel was a suspension of finely ground coal in water it was not unreasonable to assume that in a furnace environment the water would vaporize leaving the coal to be burned in a configuration similar to that of a pulverized coal furnace. If this were the case, then the combustion properties would be influenced mainly by the particle size distribution of the suspended coal and not the atomization properties of the suspension. However, extensive testing, in particular tests comparing micronized coal water fuels with pulverized coal combustion of micronized coal showed that 
- Carbon burnout was generally poorer for coal slurry combustion than pulverized coal combustion in the same furnace configuration. 1,2

- Coal slurry combustion efficiency could be improved significantly by extensive heating of the fuel prior to atomization including explosive atomization where the fuel was heated under pressure to temperatures above $100^{\circ} \mathrm{C} .3$

- $\quad$ Analysis of particulate fly ash indicated that coal in the droplets of coal water fuel tended to agglomerate in a furnace environment. Hence, atomization droplet size was a better indicator of combustion times than coal particle size distribution. This conclusion was also reinforced by single droplet experiments. 4

These studies concluded that atomization of a coal water fuel was of prime importance in determining the combustion of the fuel in a furnace.

\section{FACTORS AFFECTING ATOMIZATION}

The factors affecting atomization of coal water slurries may be divided into two categories: physical properties of the coal water slurry and mechanical properties of the burner-nozzle configuration. This program concentrated on the physical properties of the coal water slurry.

\section{Physical Properties of Coal Water Slurries}

The study of the deformation and flow of a suspension is defined as suspension rheology and is the single most important method of characterizing a coal slurry. This analysis can be conveniently broken up into three areas, all of which can impact atomization properties.

\section{Viscometric Properties}

It has been demonstrated that it is possible to describe viscometric properties of dense coal slurries by treating them as mechanical continua following the rheological behavior of a power law fluid.

$$
\tau=\mathrm{k} \dot{\gamma} \mathrm{n}
$$


where

$$
\begin{aligned}
& \mathfrak{\tau}=\text { shear stress }(\mathrm{Pa}) \\
& \mathrm{k}=\text { consistency index } \\
& \dot{\gamma}=\text { shear rate }(\mathrm{s}-1) \\
& \mathrm{n}=\text { power law index }
\end{aligned}
$$

The power law index (n) is a measure of the deviation from Newtonian behavior of the fluid, i.e. $\mathrm{n}=1$ for a Newtonian fluid, $\mathrm{n}<1$ for a pseudoplastic system, and $\mathrm{n}>1$ for a dilatant system.

In some cases a stress must be overcome to obtain fluidity. This yield stress requires a modification of Eq. 1 where:

$$
\tau-\tau_{0}=\mathrm{k} \dot{\gamma} \mathrm{n}
$$

The apparent viscosity of the system is obtained by evaluating $\mathrm{d} \tau / \mathrm{d} \gamma$ at a specific shear rate, $\dot{\gamma}$.

In programs conducted at Adelphi University and other institutions, the rheology of coal slurries tested have been described as power law behavior. 5,6 It is important to note that the power law is only valid in the shear rate range over which it was measured and cannot generally be extrapolated to other shear rate regions.

A wide range of variations in coal slurry viscometric properties have been seen including: Newtonian, pseudoplastic, dilatant, and yield power law. The type of behavior can vary depending upon coal properties, type of dispersants added to the suspension, type of chemicals added to decrease sedimentation of the coal, shear rate range, and temperature. Coal slurries have also demonstrated time dependent effects including decreasing viscosity as a function of time under shear (thixotropy) and increasing viscosity as a function of time under shear (rheopexy).

Coal water slurries are classified as suspension concentrates. Suspension concentrates are solid in liquid dispersions in which the mean particle size exceeds colloidal dimensions, i.e. greater than one micron. These coarse suspension concentrates differ from colloidal suspensions in that 
upon standing, particles will settle to the bottom of a container due to gravity, whereas colloidal suspensions are kept suspended by thermal agitation and Brownian motion.

Suspension concentrates of coal are very complex systems. Van der Waals attractive forces promote flocculation and aggregation of the particles. These attractive forces can be opposed by electrical forces of repulsion caused by a charge structure surrounding individual coal particles and steric effects due to adsorbed macromolecules which lead to an increase in free energy when particles approach each other. The stability of a suspension concentrate against sedimentation of particles is a measure of the structure of the system and has been extensively studied for coal water slurries. The degree of structure formation such as agglomeration, flocculation, and the strength of the interparticle binding forces is manifested by the rheological properties of the system under shearing conditions.

A key parameter in describing a suspension concentrate is the particle concentration or the volume fraction of solid particles, $\phi$. A prime objective in developing an understanding of the suspension rheology is the relative viscosity, $\eta_{\mathbf{r}}$, defined as the ratio of the viscosity of the suspension, $\eta_{\mathrm{S}}$, to the viscosity of the suspending fluid, $\eta \mathrm{f}$. For coal slurries at room temperature, $\eta f=1$. Therefore, $\eta_{r}$ and $\eta_{S}$ may be used interchangeably. For very dilute systems, $(\phi<0.1)$ particle-particle interactions can be neglected and it is possible to develop a theory describing $\eta$ by treating the interactions between the fluid and the individual particles. The result is

$$
\eta=1+K_{1} \phi
$$

where $\mathrm{K}_{1}$ is a constant dependent upon particle shape and is equal to 2.5 for hard spheres. As concentration $(\phi)$ increases, particle-particle interactions become important.

Including binary particle interactions adds a term $\mathrm{K}_{2} \phi^{2}$ to Equation 1-3. Multiparticle interactions between $n$ particles introduces terms in the series such $K_{n} \phi^{n}$. At very high concentrations, collective effects could become important, therefore, a simple power series description will not suffice. 
A general approach to treat complex systems such as concentrated suspensions is to assume that macroscopic behavior results from some average of material properties on the microscopic scale. However, many situations arise where large scale properties differ from simple superposition of small scale ones. Examples include: behavior near phase boundaries, superconductivity of materials, vibrations of lattices, ferromagnetism, etc. At the macroscopic level, collective properties are often observed which can be entirely new ones in which microscopic details are completely overshadowed. In fact, these collective properties can appear universal, such as bulk properties of disperse systems, hindered settling of suspensions, and pseudoplastic behavior, etc.

The simple iterative approach suggested previously which develops a power series in $\phi$ to describe $\eta$ would not be sufficient to describe a dense suspension. Under flow conditions, the scale of heterogeneity of suspensions can change due to structural modifications such as formation and breakup of agglomerates of dispersed particles. Flow in a pipe will cause concentration inhomogeneities for $\phi$ greater than a few percent.

For highly concentrated suspensions which are the main focus in the study of coal water fuels, phenomenological approaches have been developed. In the phenomenological approach, a relation between bulk properties, viscosity, $\eta$, and volume concentration, $\phi$, is obtained. ${ }^{7}$ The shear viscosity will be infinite at some value called the maximum packing, $\phi \mathrm{m}$. At this volume density of particles, the suspension will not flow under the stresses typical in pumping and flow of the suspensions. Therefore,

$$
\eta=\mathrm{F}\left(\phi, \phi_{\mathrm{m}}\right)
$$

such that

$$
\eta \rightarrow \infty \text { as } \phi \rightarrow \phi_{\mathrm{m}}
$$

Adelphi University has researched these phenomenological models and utilized one based on the following principles:

- The viscosity at typical pumping shear rates of $100 \mathrm{~s}^{-1}$ depends only on $\phi$ and $\phi \mathrm{m}$. 
- The flow pattern in typical flow situation as pipe flow consists of a concentrated dense core with a particle depleted outer core. This pattern has been observed in slurry flow.

- The functional relationship between $\eta, \phi$ should minimize viscous energy losses during flow.

- The maximum packing, $\phi \mathrm{m}$, includes structural information such as the geometric placement of particles which maximizes $\phi \mathrm{m}$ by smaller particle filling voids present in the loose packing structure of larger particles.

Application of these principles leads to a unique relation of the form:

$$
\eta=F\left(\phi / \phi_{m}\right)
$$

where

$$
\eta \rightarrow \infty \text { as } \phi / \phi_{\mathrm{m}} \rightarrow 1
$$

and

$$
\phi / \phi_{\mathrm{m}}=\text { relative packing fraction }
$$

Adelphi developed a well-defined method of computing $\phi_{m}$ from a given particle size distribution of suspended particles. In addition, this theory allows the development of particle size distribution functions which can increase $\phi_{\mathrm{m}}$ to a value greater than 0.90 . This model has been applied to the analyses of hundreds of coal slurries including an extensive development program for the Electric Power Research Institute. This model has the potential of describing non-Newtonian rheological behavior of suspension concentrates and the effect of coal surface properties by incorporating structural information in the maximum packing density. 8

Qualitative arguments can be made relating model parameters and structure. At rest, the structure of a suspension concentrate results from association of particles. The structure formed will depend on the history of the material and the interparticle forces. Increasing the shear stress will breakdown the structure either immediately or after some threshold stress. Since $\eta$ will decrease as $\phi / \phi \mathrm{m}$ decreases, this leads to pseudoplastic behavior or yield pseudoplastic behavior. As the shear rate increases, reversible breakup of the structure occurs such as breakup of 
aggregates and agglomerates, orientation of individual structures and deformation of structural units. However, certain systems such as highly concentrated suspensions of rigid particles can exhibit a shear induced build up of structure leading to a loosened packing structure, an increase in $\phi / \phi \mathrm{m}$, an increase in viscosity, and therefore, dilatant behavior. This decrease in $\phi \mathrm{m}$ may result from shear induced flocculation due to the increased particle collision frequency at higher shear rates.

An important property for atomization is the change in viscosity from pumping shear rates $\left(\sim 100 \mathrm{~s}^{-1}\right)$ to atomization shear rate $\left(\sim 5000 \mathrm{~s}^{-1}\right.$ or higher $)$. A coal water slurry with a power law index of 1.3 may experience a four or five fold increase in viscosity when shear is increased from the pumping to the atomization range. In addition, coal water slurries could become dilatant at high shear even if Newtonian at low shear. Conversely, a dilatant coal water slurry at low shear could become more Newtonian at high shear. (This is more generally the case.) Therefore, in determining whether a coal water slurry is acceptable for a combustion application such as gasifier feedstock, the entire range of shear rates must be evaluated and any model must take this into account.

Attempts to date to correlate slurry viscosity with atomization have not been successful. For a slurry utilizing a specific coal, reducing viscosity by dilution generally improves atomization. However, for two different slurries there are cases where the one which had the higher viscosity, as measured in a rotational viscometer at $100^{-1}$, atomized better. This may be due in part to the difficulty in extrapolating viscosities to the higher shear rates a slurry experiences in a nozzle. However, other factors may play a role as discussed below. 9

\section{Viscoelastic Properties}

In systems such as coal water slurries which can have internal structure, viscoelastic properties can be exhibited. These properties could affect the stability and the slurry breakup into ligaments upon exiting a nozzle orifice as well as the subsequent breakup of ligaments.

Viscoelastic behavior is measured in terms of a complex shear modulus. The complex shear modulus, $\mathrm{G}^{*}$, is defined as follows:

$$
G^{*}=\tau * / \gamma^{*}
$$


The complex stress, $\tau^{*}$, and complex shear strain, $\gamma^{*}$, are given by:

$$
\tau^{*}=\tau_{0} \text { expi }(w t+\S)
$$

and

$$
\gamma^{*}=\gamma_{\mathrm{o}} \exp (\mathrm{iwt})
$$

where:

$$
\begin{aligned}
& \gamma_{0}=\text { maximum shear strain } \\
& \tau_{0}=\text { maximum shear stress } \\
& w=\text { angular frequency } \\
& \S=\text { phase angle before the strain } \\
& i=\sqrt{-1} \\
& t=\text { time }
\end{aligned}
$$

$\mathrm{G}^{*}$ can then be given by the equation:

$$
\mathrm{G}^{*}=\tau_{0} / \gamma_{0} \exp (\mathrm{i} \S)=\tau_{0} / \gamma_{0}(\cos \S+\mathrm{i} \sin \S)=\mathrm{G}^{\prime \prime}+\mathrm{i} \mathrm{G}^{\prime}
$$

where $G^{\prime}=\left(\tau_{0} / \gamma_{0}\right) \cos \S$ is the part of the modulus that is in phase with the strain and is usually referred to as the storage modulus. $G^{\prime \prime}=\left(\tau_{0} / \gamma_{0}\right) \sin \S$ is the out-of-phase (imaginary) component of the shear modulus, referred to as the loss modulus. Thus, $\mathrm{G}^{\prime}$ is associated with the storage and release of energy during a periodic application and $\mathrm{G}^{\prime \prime}$ is associated with the dissipation of energy into heat. To obtain the time dependencies of $\mathrm{G}^{\prime}$ and G", dynamic measurements at various frequencies are required. Such measurements have frequently been carried out for viscoelastic solutions. The shear modulus can be directly related to the particle-particle interaction forces. For a fluid that is purely viscous, $\mathrm{G}^{\prime}$ is zero and the phase is $90^{\circ}$. For a purely elastic material, where energy is stored but not dissipated, $\mathrm{G}^{\prime \prime}$ is zero and the phase is $0^{\circ}$. A light oil for example would have no elastic properties, whereas an elastic material such as rubber would have $\mathrm{G}^{\prime \prime}$ close to zero and a phase close to $0^{\circ}$. 
In addition, theoretical analysis has shown that viscoelastic affects can impact the break-up of liquid jets. Viscometric measurements on the slurries that were atomized have been performed in order to determine the relationship between $\mathrm{G}^{\prime}$ and $\mathrm{G}^{\prime \prime}$ and the atomization quality.

\section{Extensional Viscosity}

The definition of extensional viscosity can be visualized in the simple case of the uniform extension of a cylinder of an incompressible Newtonian fluid along its axis. In a cylindrical coordinate system where $\mathrm{z}$ is oriented along the cylinder axis and the radial dimension ir $\mathrm{r}$, we may write the following for the case of symmetry about the $\phi$ direction:

$$
\begin{aligned}
& \sigma_{\mathrm{rr}}=-\mathrm{p}+2 \mu \frac{\delta \mathrm{v}_{\mathrm{r}}}{\delta \mathrm{r}} \\
& \sigma_{\phi \phi}=-\mathrm{p}+2 \mu \frac{\mathrm{v}_{\mathrm{r}}}{\mathrm{r}} \\
& \sigma_{\mathrm{zz}}=-\mathrm{p}+2 \mu \frac{\delta v_{\mathrm{z}}}{\delta \mathrm{z}}
\end{aligned}
$$

For the stress equation where $\mathrm{p}$ is the hydrostatic pressure, and $\sigma_{\mathrm{rr}}, \sigma_{\phi \phi}$, and $\sigma_{\mathrm{zz}}$ are the extra stresses normal to planes perpendicular to the $r, \phi$, and $z$ axis, respectively. The equation of continuity for an incompressible fluid is given by

$$
\frac{v_{r}}{r}+\frac{\delta v_{r}}{\delta r}+\frac{\delta v_{Z}}{\delta z}=0
$$

Note that

$$
\sigma_{\mathrm{rr}}+\sigma_{\phi \phi}=-2 \mathrm{p}+2 \mu\left(\frac{\mathrm{v}_{\mathrm{r}}}{\mathrm{r}}+\frac{\delta \mathrm{v}_{\mathrm{r}}}{\delta \mathrm{r}}\right)=-2 \mathrm{p}-2 \mu \frac{\delta \mathrm{v}_{\mathrm{z}}}{\delta \mathrm{z}}
$$

but since for equilibrium, $\sigma_{\mathrm{rr}}=\sigma_{\phi \phi}=0$, we obtain 


$$
p=-\mu \frac{\delta v_{z}}{\delta z}
$$

Combining Eq. (1-12) and Eq. (1-15) to eliminate p yields

$$
\sigma_{\mathrm{zZ}}=3 \mu \frac{\delta \mathrm{v}_{\mathrm{z}}}{\delta \mathrm{z}}=\mathrm{n}_{\mathrm{e}} \frac{\delta \mathrm{v}_{\mathrm{z}}}{\delta \mathrm{z}}
$$

where $n_{e}$ is defined as the extensional viscosity, which is $3 \mu$ for a Newtonian fluid.

\section{MODELING THE ATOMIZATION PROCESS}

Atomization, which is the breakup of a liquid jet into a large number of small droplets, occurs due to the impact of the jet with an air stream at large relative velocities. The mechanical factors alluded to previously affect the aerodynamics of the impinging air stream and the hydrodynamics of the initial fuel jet structure. The jets are broken up into ligaments or segments of fluid which are further broken up into a large number of smaller drops of varying size. In any attempt to model this process, both the hydrodynamical and statistical nature of the process must be taken into account. For Newtonian liquids such as fuel oils correlations have been developed between average properties, such as the Sauter mean diameter (SMD), and dimensionless numbers, such as the Reynolds number (Re), Weber number (We), and the Obnesorge number ( $\mathrm{Z}$ ), which is $\sqrt{\mathrm{We}} / \mathrm{Re}$, as well as the air to fuel ratio. The specific way in which these factors combine depends on the mechanical configuration of the atomizer being considered.

\section{PROGRAM GOALS AND OBJECTIVES}

As has been demonstrated, the complexity of coal slurries had prohibited obtaining a reasonable understanding of coal slurry atomization. The complex rheological behavior of many types of coal slurries requires an extensive characterization for each type of coal, each type of coal particle size distribution, chemical additive composition, and coal concentration. The relationship of these properties to the atomization process was therefore unclear and the ability to make a prediction as to whether a specific slurry would atomize well or whether a specific coal was suitable for combustion applications does not exist unless extensive testing is performed for each slurry. The overall objective of this program was to develop a phenomenological model for 
coal slurry atomization, which has the capability of distinguishing atomization properties for different coal slurries. In order to avoid complications due to mechanical factors, the model was developed for one type of air blast atomizer configuration. Subsidiary technical objectives included:

- Ascertain the effect of physio-chemical properties of coal slurries on atomization.

- Ascertain the statistical influence on coal slurry atomization.

- $\quad$ Predict the atomized drop size of a coal slurry from a few basic coal and slurry properties.

In the next section the methodology used for the development of a phenomenological model of coal slurry atomization is discussed.

\subsection{METHODOLOGY}

In order to accomplish the objectives of this program, a methodology was developed which included the following components:

- Sample selection and preparation including simulated fluids and coal water slurries.

- Determination of rheological properties including viscometric, extensional, and dynamic (oscillatory) flow properties.

- Determination of atomization properties as a function of fluid type and air/fuel ratio.

- Phenomenological theoretical analysis of factors affecting atomization.

- Analysis of results in terms of the phenomenological theory.

The various aspects of the methodology will be discussed separately.

\section{Sample Selection and Preparation}

Three sample fluids were prepared in order to examine the effect of varying rheological properties. One sample consisted of a $90 \%$ solution of corn syrup in water which yielded a high 
viscosity Newtonian fluid. Another sample consisted of a $70 \%$ corn syrup and $0.5 \%$ xanthan gum in water solution in order to add an elastic component and a yield point. The third fluid sample consisted of a $60 \%$ corn syrup and $1 \%$ xanthan gum designed to increase the elastic component. In addition, three coal slurries using an Illinois coal were prepared with approximately $60 \%$ solids and $0.20 \%$ surfactant and $0.5 \%$ xanthan gum. Coal solids content varied from $56-61 \%$ in order to examine the physical properties of the coal slurries.

Viscometric Properties

Moderate shear $\left(1-400 \mathrm{~s}^{-1}\right)$ viscometry was performed using a StressTech rheometer with a bob and cup measuring system. High shear extrusion rheometry was accomplished using the Adelphi high shear viscometer consisting of a sample chamber, pressure transducers and extension tube. Typical shear rates attainable were in the 1000-5000 s-1 range.

\section{DETERMINATION OF EXTENSIONAL FLOW PROPERTIES}

\section{Theoretical Analysis of Contracting Flow}

The extensional viscosity was determined in this project by analyzing contracting flow. This approach was pioneered by Bindings ${ }^{1}$ and consists of extracting data from various regions of fluid flowing in a contracting flow geometry. A theoretical derivation is presented below with a presentation of the various pressure drops occurring in an extensional viscometer based on contracting flow. In particular, by using energy considerations the meaning of each pressure drop is clearly delineated.

\section{Energy Dissipation and Extensional Flow}

The total kinetic energy of an incompressible fluid is given by:

$$
E_{\text {Kin }}=\frac{1}{2} \rho \int \mathrm{v}^{2} \mathrm{dV}
$$

where $\rho$ is the fluid density and $v$ is the fluid velocity at a point $x=(x, y, z)$ at time $t$. Subscript notation will also be used for coordinates, i.e. $x_{1}=x, x_{2}=y, x_{3}=z$, $\mathrm{x}=(\mathrm{x}, \mathrm{y}, \mathrm{z})=\mathrm{x}_{\mathrm{i}}, \mathrm{i}=1,2,3$. 
Taking the time derivative of the energy yields

$$
\frac{\mathrm{d}}{\mathrm{dt}} \mathrm{E}_{\mathrm{Kin}}=\rho \int\left(\frac{\delta \mathrm{v}_{\mathrm{i}}}{\delta \mathrm{t}}\right) \mathrm{v}_{\mathrm{i}} \mathrm{dV}
$$

where summation of repeated indicies is implied (Einstein summation convention).

The Navier Stokes equation is

$$
\delta \mathrm{v}_{\mathrm{i}} / \delta \mathrm{t}=-\mathrm{v}_{\mathrm{k}} \frac{\delta \mathrm{v}_{\mathrm{i}}}{\delta \mathrm{x}_{\mathrm{k}}}-\frac{1}{\rho} \frac{\delta P}{\delta x_{i}}+\frac{1}{\rho} \frac{\delta \sigma_{i k^{\prime}}}{\delta x_{k}}
$$

where $\mathrm{P}$ is the hydrostatic pressure and $\sigma \mathrm{i} \mathrm{k}^{\prime}$ is the extra stress tensor.

Using Eq. $2-2$ in Eq. $2-1$ and the fact that $\nabla * \mathrm{v}=0$ and

the identity $\nabla *\left(v\left(\frac{1}{2} v^{2}+\frac{P}{\rho}\right)\right)=\nabla * v\left(\frac{1}{2} v^{2}+\frac{P}{\rho}\right)+v * \nabla\left(\frac{1}{2} v^{2}+\frac{P}{\rho}\right)$ yields

$$
\frac{\mathrm{d}}{\mathrm{dt}} \mathrm{E}_{\mathrm{Kin}}=-\int \mathrm{dV} \nabla *\left[\rho \mathrm{v}\left(\frac{1}{2^{v}} 2+\frac{\mathrm{P}}{\rho}\right)-\mathrm{v}^{*} \sigma^{\prime}\right]-\sigma_{\mathrm{i}} \mathrm{k}^{\prime} \frac{\delta \mathrm{v}_{\mathrm{i}}}{\delta \mathrm{x}_{\mathrm{k}}} \mathrm{dV}
$$

where $v^{*} \sigma^{\prime}=v_{i} \sigma_{i k}{ }^{\prime}$ and $\nabla *\left(v^{*} \sigma^{\prime}\right)=\frac{\delta v_{i}}{\delta x_{k}} \quad \sigma_{i k}{ }^{\prime}+v_{i} \frac{\delta \sigma_{i k}{ }^{\prime}}{\delta x_{k}} \quad$ have been used.

Using the divergence theorem transforms Eq. 2-3 into

$$
\left.\frac{\mathrm{d}}{\mathrm{dt}} \mathrm{E}_{\mathrm{Kin}}=-\int_{\mathrm{S}} \mathrm{dA}\right) \mathrm{n}\left[\rho \mathrm{v}\left(\frac{1}{2^{\mathrm{V}}} 2+\frac{\mathrm{P}}{\rho}\right)-\mathrm{v}^{*} \sigma^{\prime}\right]-\int_{\mathrm{v}} \sigma_{\mathrm{i}} \mathrm{k}^{\prime} \frac{\delta \mathrm{v}_{\mathrm{i}}}{\delta \mathrm{x}_{\mathrm{k}}} \mathrm{dV}
$$

where the first integral is over a close surface $S$ bounding the volume $V$ and $n$ is a unit normal outward to the surface dA on S. Equation 2-4 was applied to steady flow through a contracting region shown in Figure 1. 


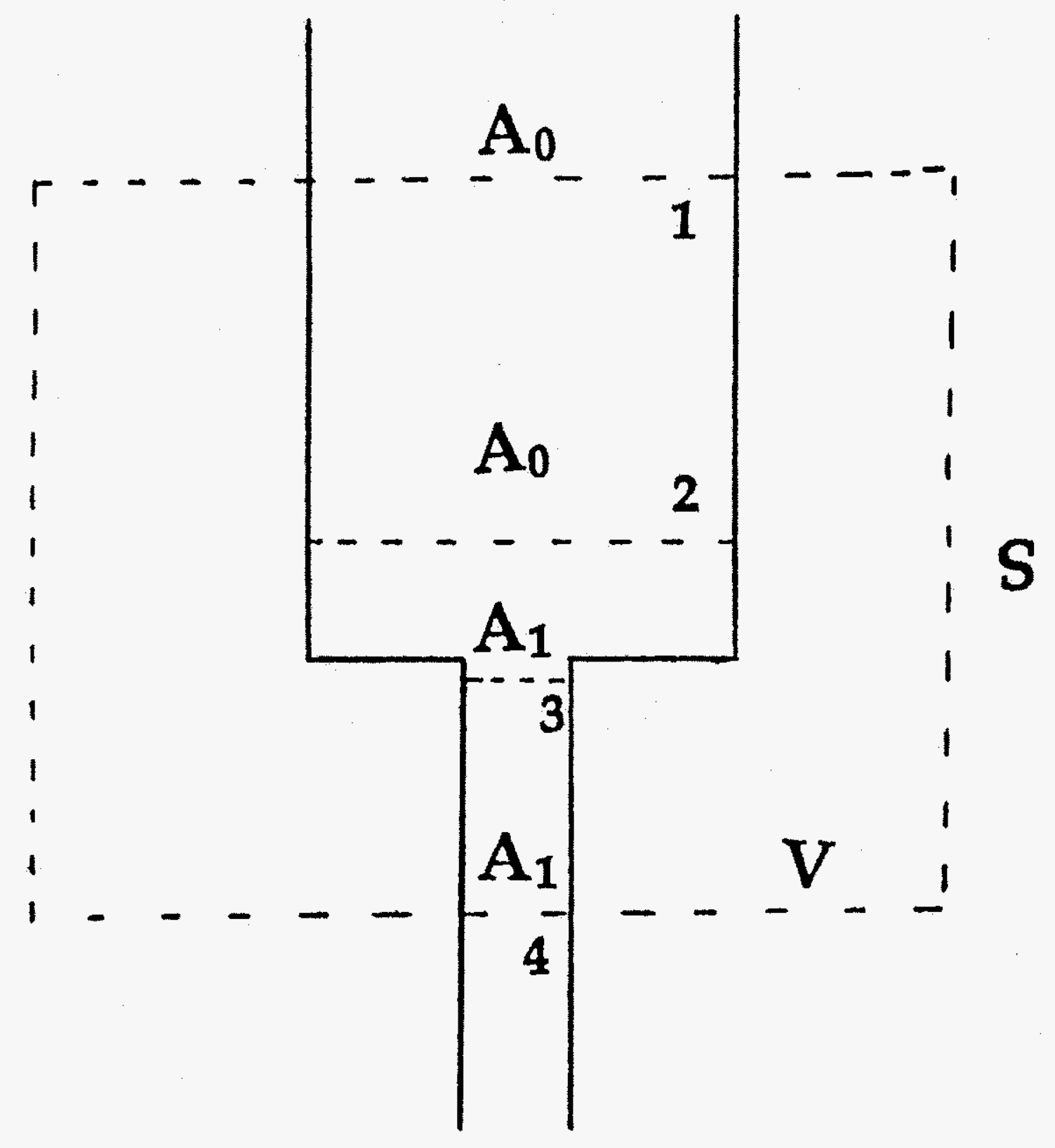

Figure 1. Contracting Flow Geometry 
The surface integral will vanish on the sides of the cylinders in Figure 1 since the velocities vanish there. Assuming that steady Poiseuille flow has been established for 1-2 and 3-4 on the cross sectional area $A_{0}$ at 1 and $A_{1}$ at 4 only $v_{3}$ exists and $n$ is the 3 direction so that the term $v$ $\sigma^{\prime *} \mathrm{n}$ becomes $\mathrm{v}_{3} \sigma_{33}{ }^{\prime}$ which is zero. For steady flow, $\frac{d}{d t} E_{K i n}=0$ since the velocity distribution is independent of time. Eq. (4) thus gives

$$
\begin{aligned}
0= & +\int_{1} \mathrm{dA} \rho \mathrm{v}\left(\frac{1}{2^{\mathrm{v}}}{ }^{2}\right)+\mathrm{P}_{1} \int_{1} \mathrm{dAv}-\int_{4} \mathrm{dA} \rho \mathrm{v}\left(\frac{1}{2^{\mathrm{v}}}{ }^{2}\right)-\mathrm{P}_{4} \int_{4} \mathrm{dAv} \\
& -\int_{\mathrm{v}} \sigma_{\mathrm{i}}{ }^{\prime} \frac{\delta \mathrm{v}_{\mathrm{i}}}{\delta \mathrm{x}_{\mathrm{k}}} \mathrm{dV}
\end{aligned}
$$

Now $\int_{1} \mathrm{dAv}=\mathrm{Q}_{1}=\mathrm{Q}_{4}=\int_{4} \mathrm{dAv}=\mathrm{Q}$ the volumetric flow rate.

Eq. 5 can be rewritten $\left(\mathrm{P}_{1}-\mathrm{P}_{4}\right) \mathrm{Q}=\Delta(\mathrm{KE}) \mathrm{Q}+\int_{\mathrm{v}} \sigma_{\mathrm{i} k} \mathrm{k}^{\prime} \frac{\delta \mathrm{v}_{\mathrm{i}}}{\delta \mathrm{x}_{\mathrm{k}}} \mathrm{dv}$

where $\Delta(\mathrm{KE})=\left(\frac{1}{2} \rho \mathrm{v}^{2}\right)_{4}-\left(\frac{1}{2} \rho \mathrm{v}^{2}\right)_{1}$

is the change in the value of the kinetic energy density averaged over cross sectional area, i.e.

$$
\int d \operatorname{Arv}\left(\frac{1}{2} v^{2}\right)=Q\left(\frac{1}{2} \bar{r}^{2}\right)
$$

The volume integral represents the viscous energy dissipation and may be broken into Poiseuille pressure drops between 1-2 and 3-4 and the pressure drop across the contraction as flows. 


$$
\int_{\mathrm{v}} \sigma_{\mathrm{i}} \mathrm{k}^{\prime} \frac{\delta \mathrm{v}_{\mathrm{i}}}{\delta \mathrm{x}_{\mathrm{k}}} \mathrm{dV}=\int_{\mathrm{v} 1-2}+\int_{\mathrm{v} 2-3}+\int_{\mathrm{v} 3-4}=\Delta \mathrm{P}_{12} \mathrm{Q}+\Delta \mathrm{P}_{23} \mathrm{Q}+\Delta \mathrm{P}_{34} \mathrm{Q}
$$

Defining $\Delta \mathrm{P}_{23}=\Delta \mathrm{P}_{\mathrm{C}}$, the final equation for pressure drops becomes

$$
\left(\mathrm{P}_{1}-\mathrm{P}_{4}\right)=\Delta \mathrm{P}_{\mathrm{T}}=\Delta \mathrm{KE}+\Delta \mathrm{P}_{12}+\Delta \mathrm{P}_{34}+\Delta \mathrm{P}_{\mathrm{C}}
$$

It is the study of $\Delta \mathrm{P}_{\mathrm{C}}$ from Eq. 2-6 that yields information on contracting flow. The basic equation is

$$
\mathrm{E}_{\mathrm{C}}=+\int_{c} \sigma_{i \mathrm{k}}{ }^{\prime} \frac{\delta \mathrm{v}_{\mathrm{i}}}{\delta \mathrm{x}_{\mathrm{k}}} \mathrm{dV}=+\frac{1}{2} \int_{\mathrm{c}} \sigma_{\mathrm{i}} \mathrm{k}^{\prime}\left(\frac{\delta \mathrm{v}_{\mathrm{i}}}{\delta \mathrm{x}_{\mathrm{k}}}+\frac{\delta \mathrm{v}_{\mathrm{k}}}{\delta \mathrm{x}_{\mathrm{i}}}\right) \mathrm{dV}=\mathrm{Q} \Delta \mathrm{P}_{3}
$$

The integral in Eq. 2-7 is over the contracting region 2-3.

As an example of applying the energy dissipation integral consider a tube of radius $\mathrm{R}$ and length $\mathrm{L}$ through which flows a fluid of viscosity $\eta$. Using the standard velocity distribution and applying Eq. 2-4 yields

$$
\Delta P Q=\frac{8 \eta Q^{2} L}{\pi R^{4}} \quad \text { or } \quad \frac{\Delta P}{L}=\frac{8 \eta Q}{\pi R^{4}}
$$

which is the result for Poiseuille flow.

It can be shown that for any fluid the flow field is such as to minimize the energy dissipation in a volume, V. ( see A.M. Freudenthal and H. Geisinger in the Encyclopedia of Physics, Volume VI pages 251-256.)

\section{Adelphi Extensional Viscometer}


The Adelphi extrusion rheometer was modified to provide extensional flow. The viscometer is comprised of a testing chamber approximately 2 feet in length and 2 inches in diameter, followed by a $15^{\prime \prime}$ tube of diameter 0.19 inches. The diameter ratio of $10: 1$ provides adequate convergence for extensional effects. The sample is placed in the testing chamber. The testing chamber cover is fastened to the chamber wall and extrusion pressure is adjusted to an initial pressure. Samples are extruded and weighed and flow rates determined. The pressure is increased as determined by sample viscosity. Characteristic pressure versus shear rate curves are drawn and evaluated. From the resultant curve, the power law index, $n$, and the viscosity are determined. The extensional viscosity is determined using Binding's ${ }^{1}$ analysis which subtracts the pressure losses in the extrusion tube which is viscometric in nature, leaving data on the entry pressure to the contraction which is directly related to the extensional viscosity.

In order to determine the effectiveness of the extensional viscometer, a verification was made using Binding's ${ }^{1}$ analysis to determine power law behavior of extensional viscosity. Figure 1 shows a $\log$ plot of the entry pressure vs. flow rate. Analysis indicates a slope of 1.2 consistent with the Newtonian calibration fluid.

1 D.M. Binding, J. Non-Newtonian Fluid Mech., 27 (1988) 173-189.

Measurement of Extensional Viscosity

The schematic diagram, Figure2, describes the relevant regions of the extensional viscometer. The relevant pressure drop for determining the extensional viscosity is the viscous energy dissipation in the contracting region $\Delta \mathrm{P}_{\mathrm{C}}$. This can be determined by measuring the entry pressure.which includes the energy dissipation and the effect of the change of kinetic energy due to contraction.

$$
\Delta \mathrm{P}_{\mathrm{e}}=\Delta \mathrm{P}_{\mathrm{C}}+\Delta \mathrm{KE}=\Delta \mathrm{P}_{1,4}-\Delta \mathrm{P}_{\mathrm{A}}-\Delta \mathrm{P}_{\mathrm{B}}
$$

Initial measurements of $\Delta \mathrm{Pe}$ were taken utilizing a Newtonian motor oil of known viscosity, a differential pressure transducer between regions 3 and 4 and utilizing the gas pressure reading for 
$P_{1}$ and extrapolating Poiseuille flow from 3-4 to include the full length of the smaller diameter tube to obtain $\Delta \mathrm{P}_{\mathrm{B}}$. The initial results were unsatisfactory in that there wasn't sufficient accuracy to obtain reliable data on $\Delta \mathrm{P}$. It was then decided to investigate the possibility of utilizing a pressure transducer between regions 1 and 4 as well as between regions 3 and 4 . To test this approach, the existing pressure transducer was used to obtain $\Delta \mathrm{P}_{1,4}$ and $\Delta \mathrm{P}_{3,4}$ calculated using the Poiseuille pressure drop with the known viscosity oil. The results for $\Delta \mathrm{P}_{1,4}$ and $\Delta \mathrm{P}_{3,4}$ are shown in Table 1 including the value of the wall shear stress, $\tau_{\mathrm{W}}$, defined as

$$
\frac{\mathrm{D} \Delta \mathrm{P}_{34}}{4 \mathrm{~L}_{34}}
$$

where $L$ is the effective length of the small tube with diameter $D$.

Except for the beginning low flow rate, the results are satisfactory and are plotted in Figure 2. The dimensionless variable, $\frac{\Delta \mathrm{P}_{\mathrm{e}}}{2 \tau_{\mathrm{W}}}$ is shown and seems to be a linear function (as expected in Newtonian flow) of the Reynolds number $\operatorname{Re}=\frac{\rho V D}{\mu}$ where $\rho$ and $\mu$ are the density and viscosity of the fluid, $\mathrm{V}$ is the average velocity and $\mathrm{D}$ is the pipe diameter. Based on these results, another pressure transducer was installed in regions 1-4 and the extensional viscosity determined via the comparison of $\Delta \mathrm{Pe}$ for various fluids.

The quantity $\Delta \mathrm{P}_{\mathrm{C}}$ is a sum of the energy dissipation due to extensional viscosity and shear viscosity. The shear viscosity can be obtained from $\triangle \mathrm{P} 34$ in Eq. 2-8. The measurements necessary are obtained from the extensional viscometer shown in Figure 2. The results are shown in Figure 2. 


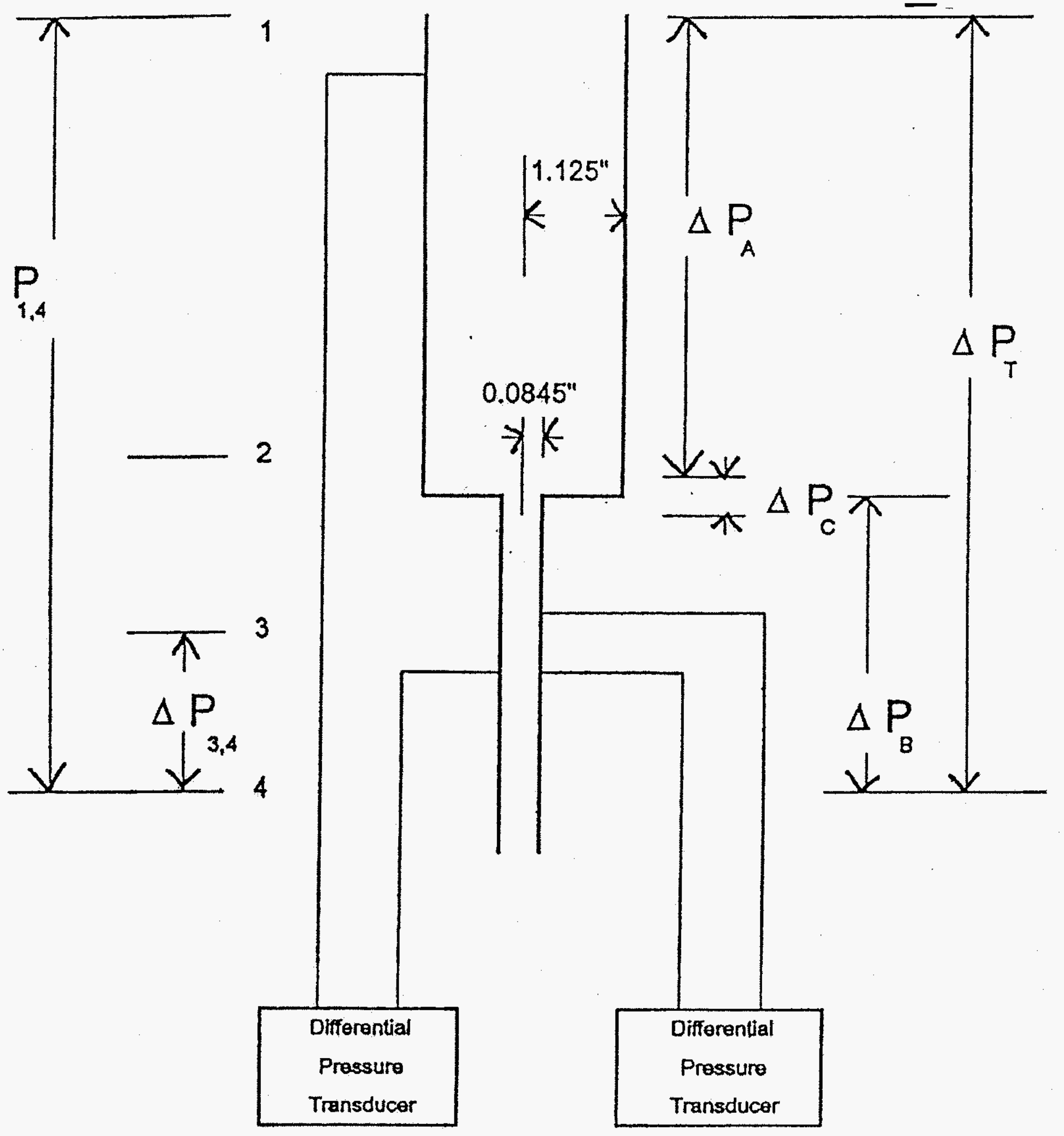

Fig 2 Extensional Viscometer Schematic 
As can be seen, $\Delta \mathrm{P}_{\mathrm{C}}$ is linear with shear rate and the slopes are proportional to the viscosities.

Therefore, an effective extensional viscosity can be defined at a specific shear rate as the slope of the tangent to the $\Delta \mathrm{P}_{\mathrm{C}}$ curve at that shear rate. This approach allows a method of analysis analogous to the determination of the shear viscosity.

\section{Viscoelastic Properties}

The Stress Tech Rheometer measures the dynamic viscosity by determining induced stresses under oscillatory flow. Both the storage modulus $G$ ' associated with energy storage and the loss modulus G" associated with viscous losses can be determined is a function of oscillatory frequency, $\omega$. The complex viscosity

$$
\mu^{*}=\sqrt{\left|G^{\prime}\right|^{2}+\left|G^{\prime \prime}\right|^{2}}
$$

\section{Atomization Studies}

For atomization studies, a progressing cavity pump ( $1 \mathrm{gal} / \mathrm{hr})$ was used to pump the fluid through a modified Delavan Aero internal mix air atomizing nozzle. A Malvern laser diffraction particle size analyzer was used to analyze the particle size distribution and air to fuel mass ratios were varied from $0.8-2.0$.

Phenomenological Theory

The theoretical analysis is based on energy consideration and incorporate various factors including viscous energy losses, kinetic energy transfers, droplet surface energies, viscoelastic and extensional flow analysis, and droplet breakup and coalescence. 
An analysis of the linearized Navier Stokes equation for atomization is utilized as a guide for the phenomenological theory.

Analysis and Conclusions

A comparison of the various factors elucidated in the theoretical studies with the data provides the basis for the conclusions.

\subsection{RESULTS}

Rheological Studies

Viscometric Data

The viscometric data includes both moderate shear (1-400 s-1) and high shear data. Moderate shear data was taken on all samples using the StressTech rheometer using the cup and bob configuration. Tablel presents the moderate shear data for the simulated fluids with a power law fit

$$
\eta=k \dot{\gamma} \quad n-1
$$

where

$$
\begin{aligned}
& \eta=\text { viscosity (mPas-s) } \\
& \mathrm{k}=\text { consistency index } \\
& \dot{\gamma}=\text { shear rate }(\mathrm{s}-1) \\
& \mathrm{n}=\text { power law index }
\end{aligned}
$$

For samples which had a yield point, i.e. those with xanthan gum added, the following equation was used for evaluation. 


$$
\tau-\tau_{0}=\mathrm{k} \dot{\gamma} \mathrm{n}
$$

where

$$
\begin{array}{ll}
\tau & =\text { shear stress }(\mathrm{dPa}) \\
\tau_{\mathrm{o}} & =\text { yield point }(\mathrm{dPa}) \\
\mathrm{k} & =\text { consistency index } \\
\dot{\gamma} & =\text { shear rate }(\mathrm{s}-1) \\
\mathrm{n} & =\text { power law index }
\end{array}
$$

Tables 1 and 2 provide the physical properties, extensional, and atomization data.

Table 1

Physical Property Results

\begin{tabular}{lcccc} 
No. Sample & \multicolumn{2}{c}{ Shear Viscosity $\left(\mathrm{mPa}^{-\mathrm{s}}\right)$} & $\begin{array}{c}\text { Ext. Visc. } \\
\left(10^{5} \mathrm{mPa}-\mathrm{s}\right)\end{array}$ & G'/G”@100HZ \\
& $100 \mathrm{~s}^{-1}$ & $5000 \mathrm{~s}^{-1}$ & & $10 \mathrm{~Pa}$ \\
1 & 386 & 300 & 4.9 & 0.49 \\
2 & 356 & 68 & 3.8 & 0.45 \\
3 & 222 & 45 & 2.12 & 0.70 \\
4 & 80 & 80 & 3.7 & $\mathrm{NA}$ \\
5 & 107 & 107 & 5.26 & $\mathrm{NA}$ \\
6 & 165 & 165 & 7.03 & $\mathrm{NA}$
\end{tabular}

1 Corn Syrup/Water 90/10

2 Corn Syrup/Water 70/30,1/2\% Xanthan

3 Corn Syrup/Water 40/60, 1\% Xanthan

4 Coal Water Slurry 56.4\%, 0.25\% A-23-S, 0.5\% Xanthan

5 Coal Water Slurry 58\%, 0.25\% A-23-S, 0.5\% Xanthan

6 Coal Water Slurry $60.7 \%, 0.25 \%$ A-23-S, $0.5 \%$ Xanthan 
Table 2

Atomization Results

Air/Fuel (mass ratio)

Sample

0.8

1.0

1.2

1.5

1.7

2.0

\begin{tabular}{rllllll}
\multicolumn{2}{l}{$\operatorname{SMD}(\mu \mathrm{m})$} & & & & \\
1 & 2 & 3 & \multicolumn{1}{l}{4} & \multicolumn{1}{l}{5} & \multicolumn{1}{l}{6} & Water \\
243 & NS & NS & 219 & 279 & 249 & 142 \\
173 & 277 & PS & 164 & 197 & 187 & 24 \\
132 & 176 & 349 & 137 & 145 & 141 & 27 \\
87 & 122 & 104 & 79 & 71 & 77 & 12 \\
53 & 121 & 54 & 47 & 41 & 55 & 11 \\
41 & 85 & 37 & 27 & 22 & 24 & NA
\end{tabular}

\subsection{THEORETICAL DISCUSSION}

The formation of drops due to wave like instabilities on the gas/liquid interface has been under intensive study for over a century. This phenomenum can be readily analyzed for several simple geometries. Atomization is a process where a stream of fluid interacts with an atomizing gas where the relative velocity between the gas and the fluid stream is large so that the stream is broken up into many droplets. In this section, atomization for a simple geometry of a cylindrical fluid jet will be analyzed by use of the Navier Stokes Equation. Following that a phenomenological model using collision theory will be presented.

This developments follows that of Levich (14). Ignoring the air viscosity and density variation air behaves as an ideal fluid. In this case the air velocity at any point in space can be characterized by a potential function $\phi(\vec{r}, t)$ where $\vec{r}$ is the position vector from

$$
\begin{aligned}
& \vec{v}=\nabla \phi=\vec{i} \frac{\partial \phi}{\partial x}+\vec{j} \frac{\partial \phi}{\partial y}+\vec{k} \frac{\partial \phi}{\partial z} \text { where } \vec{v} \text { is the velocity vector. } \\
& \vec{v}=v_{x} \hat{i}+v_{y} \hat{j}+v_{z} \hat{k}
\end{aligned}
$$

$\hat{i}, \hat{j}, \hat{k}$ are unit vectors in the $\mathrm{x}, \mathrm{y}, \mathrm{z}$ directions. 


$$
\nabla \cdot \vec{v}=\frac{\partial v_{x}}{\partial x}+\frac{\partial v_{y}}{\partial y}+\frac{\partial v_{z}}{\partial z}=\frac{\partial^{2} \phi}{\partial x^{2}}+\frac{\partial^{2} \phi}{\partial y^{2}}+\frac{\partial^{2} \phi}{\partial z^{2}}=0
$$

For air velocities less than the sound velocity, air may be considered incompressible. In cylindrical coordinates Equation $4-2$ becomes

$$
\frac{\partial^{2} \phi}{\partial z^{2}}+\frac{1}{r^{2}} \frac{\partial^{2} \phi}{\partial \theta^{2}}+\frac{1}{r} \frac{\partial}{\partial r}\left(\frac{r \partial \phi}{\partial r}\right)=0
$$

where $r=\sqrt{x^{2}+y^{2}}, x=r \cos \theta, y=r \sin \theta$

Assuming a cylindrical jet of radius a, the air velocity must meet the following conditions.

$$
\begin{aligned}
& v_{z}{ }^{A} \rightarrow V^{A} \\
& v_{r}{ }^{A} \rightarrow 0
\end{aligned} \quad r \rightarrow \infty
$$

where $\mathrm{V}^{\mathrm{A}}$ is the relative velocity between the main air stream and fuel stream.

For symmetrical waves traveling along the jet axis $(z)$ the potential for the air velocity is of the form

$$
\phi^{A}=-V_{A} Z+\phi(r)^{e^{i k z+\alpha t}}
$$

where the wave number $\mathrm{k}$ and time constant $\alpha$ are to be determined.

The equation for $\phi$ becomes

$$
\frac{1}{r} \frac{\partial}{\partial r}\left(r \frac{\partial \phi}{\partial r}\right)-k^{2} \phi=0
$$

The solution for $\phi^{A}$ is 


$$
\phi^{A}=-V_{A} z+b K o(k r)^{i k z+\alpha t}
$$

$K_{o}(k r)$ is a modified Bessel function of the $2^{\text {nd }} \mathrm{kind}$ and $\mathrm{b}$ is a constant amplitude. The relative air pressure is obtained from

$$
P_{a}=\rho^{A} \frac{\partial \phi}{\partial t}
$$

If $\zeta=\zeta_{0} e^{i k+a t}$ represents the displacement of the jet surface then $\mathrm{b}$ which occurs in equation (5) can be expressed in terms of $\zeta$ through the boundary conditions

$$
\begin{aligned}
& v_{r}{ }^{A}=v_{r} \\
& v_{z}{ }^{A}=v_{z} \quad \text { at } \mathrm{r}=\mathrm{a}
\end{aligned}
$$

where $\vec{v}_{r, z}$ refers to the jet fluid velocity. To do this the equation.

$$
\left(v_{r}\right)_{r=a}=\frac{\partial \zeta}{\partial t}+\frac{\partial \zeta}{\partial z} \frac{d z}{d t}=\frac{\partial \zeta}{\partial t}+\frac{\partial \zeta}{\partial z} v_{z} \text { is used. }
$$

To obtain $v_{r}$ and $v_{z}$ in the jet the linearized Navier Stokes Equations are used.

$$
\begin{aligned}
& \frac{\partial v_{r}}{\partial t}=\frac{1}{\rho} \frac{\partial P}{\partial r}+v\left[\frac{\partial^{2} v_{r}}{\partial z^{2}}+\frac{\partial}{\partial r}\left(\frac{1}{r}\right)\left(\frac{\partial}{\partial r}\right)\left(r v_{r}\right)\right] \\
& \frac{\partial v_{r}}{\partial t}=\frac{1}{\rho} \frac{\partial P}{\partial z}+v\left[\frac{\partial^{2} v_{z}}{\partial z^{2}}+\frac{1}{r} \frac{\partial}{\partial r}\left(r \frac{\partial v_{z}}{\partial r}\right)\right]
\end{aligned}
$$

$\nu=$ kinematic viscosity and the equation of continuity

$$
\frac{\partial v_{z}}{\partial z}+\frac{1}{r} \frac{\partial}{\partial r}\left(r v_{r}\right)=0
$$

These equations are solved by writing

$$
\begin{aligned}
& v_{r}=\nu_{r}^{0}+\nu_{r}{ }^{0} \\
& \nu_{z}=\nu_{z}^{0}+\nu_{z}
\end{aligned}
$$


where $v_{r}{ }^{0}, v_{z}^{0}$ are solutions of the ideal fluid equations $(v=0)$ obtained through the potential function $\phi$ as defined previously and $v_{r}^{\prime}$ and $v_{z}^{\prime}$ are written in terms of an additional potential function $\psi$ through

$$
\begin{aligned}
& v_{r}^{\prime}=\frac{1}{\partial} \frac{\partial \psi}{\partial z} \text { and } \\
& v_{z}^{\prime}=\frac{1}{\partial} \frac{\partial \psi}{\partial r}
\end{aligned}
$$

which automatically satisfies Equation (4-10).

Using the ideal fluid equation (Eq.4-3) in the substitution of (4-11), (4-12), (4-13) and (414) into (4-8) and (4-9) yields for $\psi$

$$
\frac{\partial^{2} \psi}{\partial r^{2}}-\frac{1}{\partial} \frac{\partial \psi}{\partial r}+\frac{\partial^{2} \psi}{\partial z^{2}}=\frac{1}{v} \frac{\partial \psi}{\partial t}
$$

for which wave like solutions are sought

$$
\psi=\psi(r) e^{i k z+\alpha t}
$$

The results which are finite as $\mathrm{v}=0$

$$
\begin{aligned}
& \psi=C_{1} I 0(k r) e^{i k z+\alpha t} \\
& \psi=C_{2} r I 1(e r) e^{i k z+\alpha t}
\end{aligned}
$$

where

$$
e^{2}=\frac{\alpha}{v}+k^{2}
$$

and $I_{0}(k v)$ and $I_{1}(e r)$ are modified Bessel functions of imaginary argument. The pressure is again given by

$$
\rho=-\rho \frac{\partial \psi}{\partial t}
$$


The displacement amplitude $\zeta_{0}$ is obtained by noting that

$$
\left.\zeta=\int d t v_{r}\right)_{r=a} d t
$$

and the balance of forces on the fluid/air interface yields the conditions

$$
\begin{aligned}
& \mu\left(\frac{\partial v_{z}}{\partial r}+\frac{\nu_{r}}{\partial_{z}}\right)_{r=a}=0 \\
& p+2 \mu \frac{\partial v_{r}}{\partial_{r}}=p^{A}+p_{\sigma}
\end{aligned}
$$

where the capillary pressure is given by

$$
p_{\sigma}=\frac{\sigma}{a^{2}}\left(1-k^{2} a^{2}\right) \zeta
$$

where $\sigma$ is the surface tension an a is the jet radius.

Using (4-15), (4-16), (4-17), (4-18), (4-19), (4-20) after obtaining $v_{r}$ and $v_{z}$ from Eq. (4-10), $(4-11),(4-12)$ and (4-13). The following is obtained relating $\alpha$ and $\mathrm{k}$ using for $k_{a}, l_{a}>1$

$$
\begin{aligned}
& I_{n}(x) \rightarrow \frac{1}{\sqrt{2 \pi z}} e^{x}, K_{n}(x) \rightarrow \sqrt{\frac{\pi}{2 x}} e^{-x} ; x>1 \\
& \alpha^{2}+2 v k^{2} \alpha\left[1-\frac{2 k l}{l^{2}+k^{2}}\right]=-\frac{\alpha k^{3}}{\rho} \frac{l^{2}-k^{2}}{l^{2}+k^{2}}+\rho^{A} \frac{k^{3}}{\rho}\left(V_{A}\right)^{2} \frac{l^{2}-k^{2}}{l^{2}+k^{2}}
\end{aligned}
$$

Equation (4-21) is the basic equation. It allows solutions for $\alpha$ real and positive which means that the wave amplitudes grow exponentially leading to breakup an atomization. 
Equation (21) is solved numerically for $\mathrm{k}_{\max }$ which yields $\alpha_{\max }$, the maximum growth rate.

Since $k_{\max }=\frac{2 \pi}{\lambda_{\max }}$ the atomized drop size can be obtained from $d=C x_{\max }$ which $C$ is a scale constant. These results are compared with simulated fluids. It is clear that the velocity dependence of the atomized drop sizes differ significantly from the theory. For this reason, a phenomenological model is developed next.

\section{PHENOMENOLOGICAL DISCUSSION}

In order to develop a phenomenological model, atomization will be treated as a collision rate process..

The air and fuel masses are related by $\dot{m}_{A}=R_{A} \dot{m}$ where $R_{A}=$ mass of air/mass of fuel. The procedure for developing a phenomenological model is to write an energy balance and then estimate each term.

$$
\Delta \dot{T}=\Delta \dot{E} \sigma+\dot{E}_{\mu}+\Delta \dot{E}_{o t h e r}
$$

In Equation (4-22) $\Delta T$ is the change in the kinetic energy, $\Delta \dot{E}_{\sigma}$ is the energy required to create the additional surface of the drops, $\Delta \dot{E}_{\mu}$ is the various viscous energy loss, and $\Delta \dot{E}_{\text {other }}$ are the energy losses such as ligament formation (extensional) overcoming any yield points and any unrecovered energy storage. In addition, drop coalescence must be taken into account after the drops are formed in the interaction volume.

The first step is to obtain the entrained drop velocity $V^{\prime}$. To do this, the momentum balance equation is written assuming all drops are moving with an entrained velocity $V^{\prime}$. A reasonable estimate is that each drop will entrain an equal volume of air. The mass rate of air entrained by the outgoing droplets of volume rate $\frac{\dot{m}}{\rho}$ is then

$$
\frac{m}{\rho} \rho A=\frac{\dot{m}_{A} \rho_{A}}{\rho R A}
$$

The momentum balance equation is then 


$$
\dot{m}_{A} V_{A}+\dot{m} V=\left(\dot{m}+\frac{\rho_{A}}{\rho R_{A}} \dot{m}_{A}\right) V^{\prime}+\dot{m}_{A}\left(1-\frac{\rho A}{\rho R_{A}}\right) V_{A}
$$

where the unentrained air continues to move with velocity $V_{A}$ the existing fuel jet has velocity $\mathrm{V}$ and $\dot{m}_{A}=R_{A} \dot{m}$ has been used.

$$
V^{\prime}=\frac{\rho_{A}}{\rho} V_{A}+V_{A} / 1+\frac{\rho_{A}}{\rho}
$$

For most air atomizers, $\frac{\rho}{\rho_{A}} V_{A} \gg V$; therefore

$$
V^{\prime}=\frac{\rho_{A}}{\rho} V_{A} \text { since } \frac{\rho_{A}}{\rho}<<1
$$

Next $\Delta \mathrm{T}$ will be calculated.

$$
\Delta \dot{T}=\frac{1}{2} \dot{m}_{A} V_{A}^{2}+\frac{1}{2} \dot{m} V^{2}-\frac{1}{2} \dot{m}_{A}\left(1-\frac{\rho_{A}}{\rho R_{A}}\right)\left(V_{A}\right)^{2}-\frac{1}{2}\left(\dot{m}+\frac{\rho_{A}}{\rho R_{A}} \dot{m}_{A}\right) V^{2}
$$

Using Equation (4-23) the equation for $\Delta \mathrm{T}$ becomes

$$
\Delta T=\frac{\dot{m} \frac{\rho_{A}}{\rho}}{2\left(1+\frac{\rho_{A}}{\rho}\right)}\left(V_{A}-V\right)^{2} \approx \frac{\dot{m}}{2} \frac{\rho_{A}}{\rho} V_{A}^{2} \text { for } V_{A} \gg V
$$

Next $\Delta \dot{E} \sigma$ is determined.

For $\dot{N}$ drops/sec, the production of surface energy is given by

$$
\Delta \dot{E}_{\sigma}=\pi \sigma \sum_{i=1}^{n} \dot{n}_{i} d_{i}^{2}
$$

where $\sigma$ is the liquid surface tension and 


$$
\sum_{i=1}^{n} \dot{n}_{i}=\dot{N}
$$

The total mass rate of drops obeys the equation

$$
\dot{m}=\rho \sum \dot{n} d_{i}{ }^{3} \frac{\pi}{6}
$$

The sauter mean diameter is defined by

$$
S=\frac{\sum \dot{n}_{i} d_{i}^{3}}{\sum \dot{n}_{i} d_{i}^{2}}
$$

This yields

$$
\Delta \dot{E}_{\sigma}=\frac{6 \sigma}{\rho} \frac{\dot{m}}{S}
$$

If all other terms by $\Delta \dot{E}_{\sigma}$ are ignored on the rhs of equation (4-22) then

$$
\begin{aligned}
& \frac{\dot{m}}{2} \frac{\rho_{A}}{\rho} V_{A}^{2}=\frac{6 \sigma}{\rho} \frac{\dot{m}}{s} \\
& S=\frac{12 \sigma}{\rho_{A} V_{A}^{2}}
\end{aligned}
$$

which agrees with Eq.(4-21) in the previous section for low viscosities.

\section{VISCOUS ENERGY LOSSES IN DROP PRODUCTION}

To obtain $\Delta \dot{E} \mu$ the following arguments are used.

The fluid exiting the nozzle is modeled by a cylinder of radius a and length $z$ on which are produced waves of wave length $\lambda$, radial amplitude $\zeta$, frequency $\omega$, and subject to the condition $\omega \lambda \sim \sqrt{\frac{\rho_{A}}{\rho}} V_{A}$ which can be derived from Equation 4-21 under the conditions of low viscosities and small drops. 


\section{Drop production from a fluid cylinder}

A wave of wavelength $\lambda$ will produce $\frac{L}{\lambda}$ drops. The change in volume as drops come off is $\Delta \Omega=\frac{L}{\lambda}\left((a+\zeta)^{2} \frac{\lambda}{2}-a^{2} \frac{\lambda}{2}\right)=a \zeta L=\zeta A$ where $A$ is the surface area of the cylinder.

Since there are $\frac{\omega}{2 \pi}$ waves $/ \mathrm{sec}$.

$$
\frac{d \Omega}{d t} \doteq \omega \Delta \Omega=\omega a \zeta L=\omega \zeta A=\frac{\dot{m}}{\rho}
$$

The viscous energy loss in producing these drops can be estimated as follows

$$
\dot{E}_{\mu}=\mu \dot{\gamma} \omega \zeta A
$$

where the shear rate is

$$
\dot{\gamma} \doteq \frac{\omega \zeta}{\lambda}=k \omega \zeta
$$

and the surface area is $\mathrm{A}=\mathrm{aL}$.

This yields

$$
\dot{E}_{\mu}=\mu \omega^{2} k A \zeta^{2}
$$

Using Eq.(4-34) and the small amplitude approximation $k \zeta \sim \beta<1$.

Using $\omega \sim \sqrt{\frac{\rho_{A}}{\rho}} \frac{V_{A}}{\lambda}$ (Eq. (4-21) for $\frac{W e}{\operatorname{Re}}<1$

$$
\Delta E \mu=\sqrt{\frac{\rho_{A}}{\rho}} \mu \frac{\dot{m}}{\rho} \frac{V_{A}}{S} \beta
$$

Adding this term to Eq.(4-22) yields 


$$
\frac{\dot{m}}{2} \frac{\rho_{A} V_{A}^{2}}{\rho}=\frac{6 \sigma}{\rho} \frac{\dot{m}}{S}+\sqrt{\frac{\rho_{A}}{\rho}} \mu \frac{\dot{m}}{\rho} \frac{V_{A}}{S} \beta
$$

which gives the equation for $\mathrm{s}$

$$
S=\frac{12 \sigma}{\rho_{A} V_{A}^{2}}+2 \sqrt{\frac{\rho_{A}}{\rho} \frac{\mu}{\rho_{A} V_{A}}} \beta
$$

This can be rewritten in terms of the Weber number $W e=\frac{\rho_{A} V_{A}^{2} a}{\sigma}$ and the Reynolds number for drops.

$$
\begin{gathered}
\operatorname{Re}=\frac{\rho V^{\prime} a}{\mu}=\rho \frac{\rho_{A}}{\rho} \frac{V_{A} a}{\mu}=\rho_{A} \frac{V_{A} a}{\mu}=\rho_{A} \frac{V_{A} a}{\mu} \\
\frac{S}{a}=\alpha\left(12 W e^{-1}+2 \beta \sqrt{\frac{\rho_{A}}{\rho}} \operatorname{Re}^{-1}\right)
\end{gathered}
$$

where $\alpha \& \beta$ are constants to afford appropriate scale. By appropriate choice of $\alpha, \beta$ very good agreement is reached between Eq.(4-38) and the solution of Eq.(4-21) for a range of viscosities $(100-300 \mathrm{cP})$.

In the limit of large viscosities $\left(\frac{W e}{\operatorname{Re}}>1\right)$ and small drops ka $>>1$ Eq. $4-21$ yields.

$$
\frac{S}{a} \rightarrow\left(\frac{\rho_{A}}{\rho}\right)^{1 / 3} \frac{Z^{2 / 3}}{W e^{2 / 3}}
$$

where $\mathrm{Z}$ (Ohnsager number) $=\frac{\sqrt{W e}}{\operatorname{Re}}$.

A interpolatory formula which reduces to (4-38) and (4-39) in the appropriate regions and agrees with Eq. 4 -21 over a range of velocities is given by

$$
\frac{S}{a}=\frac{\alpha}{W e}\left(1+\beta \frac{W e}{\operatorname{Re}} \sqrt{\frac{\rho_{A}}{\rho}}\right)^{2 / 3}=\frac{\alpha}{W e^{2 / 3}}\left(W e^{-1 / 2}+\beta \sqrt{\frac{\rho_{A}}{\rho} Z}\right)^{2 / 3}
$$


Eq.(4-39) the propagation velocity is given by

$$
\omega \lambda=\frac{\sigma^{1 / 3}}{\mu^{1 / 3}} \rho_{A}^{1 / 3} V_{A}^{2 / 3}
$$

Effects of Non-Newtonian Flow

The theoretical and the phenomenological model discussed previously must be modified to take into account the following effects

- Non-Newtonian flow characteristics

- Turbulence effects

- Drop coalescence and breakup

- Large amplitude effects

- Statistical considerations

Because the flow regime considered for producing drops involves small amplitude vibrations, the dynamic viscosity could be a better indicator of viscous losses in drop production than either the moderate shear $(1001 / \mathrm{s})$ or high shear viscosity $(>50001 / \mathrm{s})$. Using the dynamic loss viscosity, Equation 4-40 agrees with the trend in the data better than either of the shear viscosities. Another important consideration is the effect of a yield point $\tau_{0}$.

Following the approximation procedures described previously, the effect of the yield point is obtained by adding a term $\tau_{0}(\omega \zeta) A$ which represents work done against the yield stress to the viscous energy loss term. The relative importance of the yield point and the breakup forces due to air is $\frac{\tau_{0}}{\rho_{A} V_{A}{ }^{2}}$. This can be expressed as the ratio of the yield point number $Y=\frac{a \tau_{0}}{V_{A} \mu}$ and the Reynolds Number $\frac{\rho_{A} V_{A} a}{\mu}$. This has the effect of multiplying Eq.19 by a factor $\left(1-\gamma * \frac{Y}{\mathrm{Re}}\right)^{-1}$ where $\gamma$ is an adjustable constant. This effect correctly reproduced the trend of the data, which shows difficulty of atomization at the lowest air velocities for fluids with high yield points. 
The relaxation times of the fluids with viscoelastic properties are much greater than the atomization times. Therefore, energy stored in the formation of wave-like surface oscillations may not participate in the atomization process. If the dynamic complex viscosity is represented by

$\mu^{*}=\mu^{i}-i \mu^{\prime \prime}$

where $\mu^{\prime}=\frac{G^{\prime \prime}}{\omega}$ and $\mu^{\prime \prime}=\frac{G^{\prime}}{\omega}$ where $G^{\prime \prime}$ is the loss modulus and $G^{\prime}$ is the storage modulus. Elastic energy storage rate is estimated by $G^{\prime} \gamma \omega \zeta A$ where $\gamma$ is strain $\left(\frac{\dot{\gamma}}{\omega}\right)$ and $G^{\prime} \gamma=\frac{G^{\prime} \dot{\gamma}}{\omega}=\mu^{\prime \prime} \omega$ and assuming similar high frequency behavior for $\mu^{\prime}$ and $\mu^{\prime \prime}$ then the viscosity in Eq. 4-40 should be replaced by an effective viscosity $\mu_{\text {eff }}=\mu^{\prime}\left(1+\beta_{2} \frac{G^{\prime}}{G^{\prime \prime}}\right)$ where $\beta_{2}$ is a constant which pushes the theory closer to the trend of the data. The other non-Newtonian flow characteristic of interest is the extensional viscosity. Taking the ratio of the effective extensional viscosity to the shear viscosity in the extensional viscometer shows that these ratios are higher for the fluids with xanthan gum than for the approximately Newtonian fluid containing $90 \%$ corn syrup. Assuming that a certain amount of stretching by the air of the fluid ligaments occurs during atomization. as well as the stretching necessary to deform drops for drop breakup then an estimate is made for the work done in extension using the dimensional argument of $\frac{\Delta W_{e x t}}{\Delta E_{\text {air }}} \sim \theta\left\{\left(\operatorname{Re}^{e x t}\right)^{-1}\right\}$ where $\left(\operatorname{Re}^{e x t}\right)$ is an appropriate Reynolds Number.

For the length scale in $\operatorname{Re}^{\text {ext }}$ assume $1 \sim \lambda$. The velocity of the extension could be the wave propagation velocity $\sqrt{\frac{\rho_{A}}{\rho}} V_{A}$.

The extensional viscosity effect is taken into account by using $\beta_{3} \theta \mu$ where $\theta$ is an effective Deborah number $\left(\theta=\frac{\mu_{\text {ext }}}{\mu_{\text {shear } / \text { const }- \text { fow }}}\right)$ and $\beta_{3}$ is a constant. 
The net effect of adding this term to Eq.4-38 is to add a term to the effective viscosity such that $\mu_{\text {eff }}=\mu^{\prime}\left(1+\beta_{2} \frac{G^{\prime}}{G^{\prime \prime}}+\beta_{3} \theta\right)$ which is then assumed to replace $\mu$ in Eq. 4-40. There could be other effects of extensional flow if other pumps besides positive displacement pumps are used to produce the atomizing stream. High pressure drops may be required to produce ligament streams suitable for atomization.

\section{Effect of Turbulence}

Considering the high air velocities, there will be some turbulence dissipation in the impinging streams which will reduce the effective kinetic energy transferred to the atomization process which is reflected in the multiplicative factor in Eqs. 4-38 and 4-40 being much greater than one. In addition, for low viscosity fluids such as water there will be energy dissipation in the atomizing fluid of the order of

$$
\mu_{\text {urb }}(\omega) \zeta \omega A \sim \beta_{4}\left(\rho \zeta^{2} \omega\right) \zeta \omega A \beta_{4} \rho \zeta^{3} \omega^{3} A \sim \beta_{4} \rho(\zeta \omega)^{2} \sim \beta_{4} \rho\left(\sqrt{\frac{\rho_{A}}{\rho}} V_{A}\right)^{2} \frac{\dot{m}}{\rho} \sim \beta_{4} \rho_{A} V_{A}{ }^{2} \frac{\dot{m}}{\rho}
$$

where the turbulence scale is assumed to be the wave amplitude $\zeta$ and $\beta_{4}$ is an constant. This modified the elementary expression (Eq. 4-33) by multiplication of a factor of $\left(1-\beta_{4}\right)^{-1}$. This yields a more rapid fall off of the drop size for low viscosity fluids with velocity than high viscosity fluids.

\section{Drop Coalescence and Breakup}

In order to examine the effect of coalescence and breakup of drops, a drop propagation equation for an atomized spray will be developed. Assuming a unidirectional narrow spray in the $\mathrm{z}$ direction, if an infinitesimal volume of drops moves from $\mathrm{z}$ to $z+v \delta t$ in time $\delta t$ where $\mathrm{v}$ is the drop velocity, the change in density is given by

$$
\left.n(z+v \delta t, t+\delta t)-n(z, t)=\frac{\delta n}{\delta t}\right) \text { collisions } \delta t
$$

This leads to 


$$
\left.\frac{\delta n}{\delta t}+\frac{\delta n}{\delta z} v=\frac{\delta n}{\delta t}\right)_{\text {collisions }}
$$

For an equilibrium spray $\frac{\delta n}{\delta t}=0$

$$
\left.\left.\left(\frac{\delta \eta}{\delta t}\right)_{c o l l}=\frac{\delta \eta}{\delta t}\right)_{a}+\frac{\delta \eta}{\delta t}\right)_{b}
$$

$\left.\frac{\delta \eta}{\delta t}\right)_{a}$ is the effect of coalescence or agglomeration.

$\left.\frac{\delta \eta}{\delta t}\right)_{b}$ is the effect of drop breakup.

Estimate of the Coalescence Effect

As shown by Levich ${ }^{14}$ for the drop sizes of interest $(10-300 \mu \mathrm{m})$, the collision of drops due to turbulence is the main physical process responsible for drop-drop coalescence. It can be shown under these conditions, assuming all collisions result in coalescence that

$$
\left.\frac{\delta \eta}{\delta t}\right)_{a} \approx-\frac{\rho}{\rho A} \operatorname{Re}_{A}^{5 / 4} V_{A} \frac{\bar{\lambda}^{4}}{a^{2}} n^{2}
$$

where $\bar{\lambda}^{4}$ as an average of the particle (drop size) over the particle distribution.

Re, $\mathrm{A}$ is the Reynolds number for air $\sim \frac{a V_{A}}{v}$ where $v$ is the kinematic viscosity of air at the temperature of the spray environment. The quantity a is a characteristic dimension taken at the nozzle diameter. In order to estimate $\bar{\lambda}$ referencing the breakup process, the drop distribution is given by $p(x) \sim \frac{L}{\lambda}$ where $L$ is the intact fuel stream length. Perform the integral of this distribution $\left[\int_{a}^{\lambda_{m}} d p(x)\right]$ yields the following for the total 
number of drops from a breakup of a mean of size $\mathrm{L} N(L) \sim W e(L)=\rho_{A} \frac{V_{A}{ }^{2} L}{\sigma}$ since $\lambda \mathrm{m}$ is the minimum wavelength which is $\frac{\sigma}{\rho_{a} V_{a}{ }^{2}}$ for any fluid.

For $\bar{\lambda}$ as a first estimate, the values of $\mathrm{Xmax}$ for the previous sections will be used.

\section{Effect of Drop Breakup}

In order to determine the effect of drop breakup, the following approach is taken. The Navier Stokes Equation can be used to estimate the breakup frequency, $\omega_{b}$. The breakup of drops is due to the growth of wave like distributions as discussed previously. The small amplitude equation is

$$
\frac{\delta v}{\delta t}=-\frac{\nabla p}{\rho}+\nu \nabla^{2} \vec{v}
$$

For high relative air velocities, $V_{A}, \mathrm{p} \sim \rho_{A} V_{A}^{2}$.

For low viscosities,

$$
\begin{aligned}
& \frac{\delta v}{\delta t} \sim \frac{\lambda}{t_{b}{ }^{2}} \sim \frac{\rho_{A} V_{A}{ }^{2}}{\rho \lambda} \\
& \tau_{b}=\omega_{b}{ }^{-1} \sim \frac{\lambda}{V_{A}} \sqrt{\frac{\rho}{\rho_{A}}}
\end{aligned}
$$

where $\lambda$ is characteristic of drop size.

For high viscosities $\frac{\partial v}{\partial t}$ can be neglected yielding.

$$
\begin{aligned}
& \frac{v}{\lambda \tau_{b}} \sim \frac{\rho_{A} V_{A}^{2}}{\rho \lambda} \text { or } \\
& \tau_{b}=\omega_{b}{ }^{-1} \sim \frac{\mu}{\rho_{A} V_{A}{ }^{2}}
\end{aligned}
$$


the same result as derived previously. Note that if there is a yield stress $\tau_{0}$, the equation becomes

$$
\begin{aligned}
& \frac{\mu}{\tau_{b}}+\tau_{0} \sim \rho_{A} V_{A}{ }^{2} \text { or } \\
& \tau_{b}=\omega_{b}{ }^{-1} \sim \frac{\mu}{\rho_{A} V_{A}{ }^{2}-\tau_{0}}
\end{aligned}
$$

Next consider the propagation of $\mathrm{n}$ drops in a series of discrete steps along the $\mathrm{z}$ direction of length $\Delta z=\frac{v}{\omega_{b}}$ where $v$ is the drop velocity.

In a length $z$, there are $\frac{z}{\Delta z}$ steps. After moving though a distance $\Delta z$, the contribution of the breakup of particles of size $\lambda_{i}$ to the total number of drops is

$\Delta n_{i}=Q\left(\lambda_{i}\right) N\left(\lambda_{i}\right)$ where $\mathrm{Q}$ is the number of drops/breakup given by $W_{e}\left(x_{i}\right)$ in the elementary model considered here. The total contribution of all drop sizes to $n \mathrm{n}$ is given by

$$
n(z+\Delta z)=\sum \Delta n_{i}=\sum Q\left(x_{i}\right) n\left(\lambda_{i}\right)=\int Q\left(\lambda_{i}\right) \frac{a n}{a x} d x=\vec{Q}\left(\lambda_{i}\right) \vec{n}(z)=W e(\bar{\lambda}) \vec{n}(z)
$$

$$
n(z+\Delta z)=\int \frac{d n}{d x} d x=\int \frac{d n}{d x} d x+v \frac{d}{d z} \int \frac{d n}{d \lambda} \omega_{b}^{-1} d x=n+v{\bar{\omega}_{b}}^{-1} \frac{d n}{d z}
$$

The result is

$$
v \frac{d n}{d z} \approx \bar{\omega}_{b} W e(\bar{x}) n
$$

for $W e \gg>1$. The final propagation equation is given by 
$\nu \frac{d n}{d z}=-\frac{\rho}{\rho_{A}} R_{e, A} 5 / 4 V_{A} \frac{\bar{\lambda}^{4}}{a^{2}} n^{2}+\omega_{b} W e(\bar{x}) n$

Equation 4-54 can be written in the general form

$\frac{d n}{d z}=-\alpha n^{2}+\beta n$

which has the solution dropping vanishingly small terms for $z \geq 1 \mathrm{~cm}$,

$$
n=\frac{\beta}{\alpha}
$$

From mass conservation of the atomizing fuel

$n=\frac{\dot{m}}{\rho v A \bar{\lambda}^{3}}$ where $\bar{\lambda}^{3}$ is the volume mean droplet size and $A(z)$ is the cross sectional area at a distance $z$ into the spray which can be estimated by $\theta^{2} z^{2}$ where $\theta$ is the spray angle in radians. The final result for the drop size is

$$
\begin{gathered}
\bar{\lambda}^{3}=\frac{\dot{m}}{\rho(v A)} \frac{\alpha}{\beta} \text { or } \\
\lambda=\left(\frac{\dot{m}}{\rho(v A)}, \frac{\alpha}{\beta}\right)^{1 / 3}=\left(\frac{\dot{m}}{\rho_{A} V_{A} A} \frac{\alpha}{\beta}\right)^{1 / 3}
\end{gathered}
$$

The result for water using $\bar{\lambda}=K \frac{a}{W e(a)}$ where $\mathrm{K}$ is a numerical constant

$$
\lambda=a K^{\prime}\left(\frac{\rho}{\rho_{A}}\right)^{5 / 6}\left(\frac{\dot{m}}{\mu_{a}^{2}} \frac{\sqrt{a^{3} \rho_{A} \sigma}}{A}\right)^{1 / 3} W e^{-1.63}
$$

where $\mu_{a}=$ the absolute viscosity of air. 
In order to take into account the limiting drop size ${ }^{a} W e^{-1}(a)$ the critical drop size is added in yielding

$$
\lambda=S=a\left(K^{-1}\left(\frac{\rho}{\rho_{A}}\right)^{5 / 6}\left(\frac{\dot{m}}{\mu_{a}{ }^{2}} \frac{\sqrt{a^{3} \rho_{A} \sigma}}{A}\right)^{1 / 3} W e^{-1.63}+K_{2} W e^{-1}\right)
$$

The water data can be fit with approximately $90 \%$ confidence level using this equation.

\section{ALTERNATE APPROACH}

Because the breakup of drops occur in a turbulent air stream it is reasonable to us the following estimates for $Q$ in Equation (4-52). Assume a drop of size $\bar{\lambda}$ breaks up in $Q(\bar{\lambda})$ drops due to pressure variation of the turbulent air stream. Following Levich the drops will break until a critical size $\lambda_{c r} \sim K V_{A}-6 / 5$ is reached. Using the elementary mass balance for the breakup of drop of radius $\bar{\lambda}, Q$ is determined as follows.

$\bar{\lambda}^{3}=\lambda_{c r}^{3} Q(\bar{\lambda})$

yielding

$Q(\bar{\lambda})=\frac{\bar{\lambda}^{3} V_{A}^{18 / 5}}{K^{3}}$

This changes $\beta$ in Eq. $4-55$ to

$$
\beta=\widetilde{K}_{1} \frac{\omega_{b}}{V_{A}} V_{A}^{18 / 5} \vec{\lambda}^{3}
$$

where $\widetilde{K}_{1}$ is a numerical constant.

For water in air using Eq.4-49

$$
\beta_{w}=\sqrt{\frac{\rho_{A}}{\rho}} \widetilde{K}_{1} V_{A}^{18 / 5} \bar{\lambda}^{2}
$$


Using Eq. (4-49)

$$
n=\frac{\dot{m}}{\rho v A \bar{\lambda}^{3}}=\bar{K}_{1} \frac{V_{A} \frac{18 / 5}{\mathrm{Re}^{5 / 4} \bar{\lambda}^{2}}}{2}
$$

where $\bar{K}$ is a constant. Solving Eq. $4-65$ for $\bar{\lambda}$ yields

$$
\bar{\lambda}=\frac{K_{1}^{\prime}}{A} W e^{-1.68}
$$

which agrees well with the water with the adjustable constant $K_{1}^{\prime}$. The effect of the spray angle is incorporated in A as discussed previously.

The effect of fluid viscosity is taken into account by using Eq. 4-50.

The result for $\beta$ is

$$
\beta_{\nu}=\frac{\widetilde{K}_{2}}{\mu} V_{A}^{23 / 5} \bar{\lambda}^{3}
$$

Putting this into equation 4-56

$\frac{\dot{m}}{\rho v A \bar{\lambda}^{3}}=\frac{\bar{K}_{2} V_{A}^{23 / 5}}{\mu \mathrm{Re}^{5 / 4} \bar{\lambda}}$

This yields the following expression for the drop size.

$$
\bar{\lambda} \doteq \frac{K_{2}^{\prime} \sqrt{\mu}}{\sqrt{A}} W e^{-1.09}
$$

Equation 4-68 agrees with the general trend of the data using an effective viscosity and has similar velocity dependence for the drop size.

\subsection{CONCLUSIONS}

The phenomenological model describes some essential features of atomization. One is a power law dependence of the average atomized drop size with respect to the Weber 
number of the atomizing stream based on the relative velocity of the fluid and the atomizing air. The decrease in drop size with increasing Weber number is less rapid for viscous fluids and the drop size is proportional to the square root of the viscosity of the fuel for viscous fluids. Increased spray angle will also decrease the drop size. The theoretical model also describes the similar effects but the agreement with the data is better with the phenomenological model. An important difference between the two models is that the theoretical model is based on the growth of the sinusoidal disturbance on the fuel stream exiting the nozzle where the phenomenological model is statistical in nature and dominated by drop coalescence and breakup.

There are indications that the viscosity that should be used for non-Newtonian coal slurries is an effective viscosity composed of dynamic viscosity components and extensional effects. In addition, any yield point in the fuel appears to play a direct role yielding larger drop sizes in the regime of low air/fuel ratios and lower Weber number. In the theoretical model, viscous energy losses play an important role. In the phenomenological model, the effect of fluid viscosity is to increase the time required for drop breakup which reduces drop breakup effects for a fixed interaction volume as occurs in an atomizer furnace configuration. The phenomenological model provides a basis for atomizer design and a framework for incorporating non-Newtonian effects into predicting atomizer performance.

Recommended future work should include incorporation of the effects of specific distribution functions for the drop size in order to determine not only average drop sizes but distribution widths. In addition, some of the predictions of the phenomenological model (i.e. dependence of effective viscosity, spray angel effects) should be further tested for a variety of fluids with differing physical properties. 


\section{REFERENCES}

1. P.J. Winters, K.R. Olen, R.T. Bailey, "The Effects of Fuel Formulation on Atomization Characteristics of Coal-Water Mixtures", Seventh International Symposium on Coal Slurry Fuels Preparation and Utilization, May 1985, New Orleans, LA.

2. K. Gomi, et al., "Comparative Combustion Studies of Ultrafine Coal/Water Slurries and Pulverized Coal", Seventh International Symposium on Coal Slurry Fuels Preparation and Utilization, May 1985, New Orleans, LA.

3. J. Beer, et al., "Atomization of Coal-Water Fuel at Elevated Pressure, Fourteenth International Conference on Coal and Slurry Technology", April 1989, Clearwater, FL.

4. Y. Levendis, "Combustion Rates of Coal Water Slurry Droplets", 17th International Conference on Coal Utilization and Slurry Technologies, April 28 - May 1, 1992, Clearwater, FL.

5. J. Dooher, et al., "The Rheology of Coal/Water Mixtures", AIChE National Meeting, Anaheim, CA, May 1984.

6. J. Dooher, et al., "Rheology and Handling Properties of Coal Water Slurries," ASME Winter Meeting, New Orleans, LA, December 1984.

7. J. Dooher, et al., "Physio-Chemical Properties of Coal Water Gasifier Feedstock," Presented at the Fourteenth International Conference on Coal and Slurry Technology, April 1989.

8. J. Dooher, et al., "Phenomenological Modeling of the Rheological Properties of Coal Suspensions",17th International Conference on Coal Utilization and Slurry Technologies, April 28 - May 1, 1992, Clearwater, FL.

9. S. Tsai, "Atomization of Coal Water Slurry Using Twin-Fluid Jet Atomizer", FUEL 1987, Vol 66, November.

10. J. Dooher, et al., "Factors Affecting Atomization of CWF," Coal Slurry Fuels Preparation and Utilization, Orlando, FL, May 1986.

11. W. Rakitsky, "Rheological Properties of Coal Water Fuels Significant for Fine Spray Production During Combustion", Third Annual Pittsburgh Coal Conference, Pittsburgh, PA, September 1986. 
12. J. Dooher, D. Wildman, "A Comparison of Rheological and Atomization Data For Coal Water Mixtures and Liquid Mixtures", Fourteenth International Conference on Coal and Slurry Technology, April 1989, Clearwater, FL.

13. W. Schowalter, Mechanics of Non-Newtonian Fluids, First Edition 1978, Pergamon Press Inc.

14. Levich, "PhysioChemical Hydrodynamics", Prentice Hall, 1962 
PHYSICS \& ENGINEERING MAJORS FALL 2002

\section{Advisor}

1. Akhmedov, Rustam T

2. Eriksen, Carl J

3. Hodges, John D

4. Jamison, Matthew J

5. Ledetsch, Frederick $A$

6. Mofunanya,Tobechukwu C

7. Palermo, Thomas

8. Solages, Schumann

9. Uzzaman, Faiz

10. Hada, Tatsuya

11. Andrade, Dulce $M$

12. Chang, Fabio NG

13. Manov, Alexandre M

14. Milani-Baladi, Mahnaz

15. Mueller, Patricia A

16. Neal, Brian T

17. Trongone, Eric

18. Guardia, Christian

19. Mueller, Patricia A

20. Shtrepi, Jonida $M$

21. Castro, Roberto $C$

22. Gutierez, Stephen

23. Solages, Schumann

24. Derubis, Dominic

25. Hansen, Kevin R

26. Regis, John

27. Gennawey, Andrew P

28. Schoellig, Gregory J

29. Barclay, Faith

30. Bella Jr. Thomas J

31. De La Rosa, Noren A
SO

FR

FR

FR

FR

$F R$

FR

FR

FR

$\mathrm{FR}$

so

so

so

$J R$

so

so

so

$J R$

so

So

$J R$

$J R$

FR

SR

$S R$

SO

$J R$

$S R$
718-821-2845

516-867-8329

718-366-5518

516-565-5535

516-431-6672

016-165-38

631-281-5690

718-805-7649

718-459-2951

042-576-391

818-893-5364

718-321-2471

516-741-2664

516-328-1907

516-623-3175

631-473-6742

516-681-0194

$A$

Ahner, Henry

516-741-3353

516-623-3175

Della Croce, Diane

516-877-6176

Ahner, Henry

Ahner, Henry

.516-867-6258

Dooher, John

516-354-3124

718-677-5992

516-799-8056

631-289-2951

516-378-5254

516-795-6934

Ahner, Henry

Dooher, John

Ahner, Henry

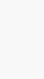

Undecided but interested in Engg. 